\title{
PICKUP AND DELIVERY PROBLEM
}

Vehicle routing problem and traveling salesman problem are classical problems in operational research; this modification of those problems consists of a transport among nodes of the communication network using cyclical routes of vehicles with a given capacity. A transportation demand is given by the place of pickup, the place of delivery and quantity of goods. The goal is to find cyclical routes of a minimal length which ensure the transport requirements. In the paper there are two models proposed for the problem, both are demonstrated on an example. The problem is based on a case study from practice.

Keywords: Pickup and delivery problem, integer programming, heuristic methods

\section{Introduction}

Traveling salesman problem and vehicle routing problem and their modifications are frequently solved practical examples. In such problems the goal is to assure pick-up or/and delivery of goods in a distribution network. While in those applications it is necessary to find cyclical routes starting and ending in a depot, in the studied problem it is required to transport goods between nodes of the network. Each requirement is specified by the pickup point, the delivery point and the amount of goods which has to be transported. In [1] and [2] the problem is called pickup and delivery problem.

In the practice time windows are often given for each node in the network and the order of pickup and delivery processes has to be defined, because the goods being loaded as the last ones will be unloaded as the first ones. The origin and the destination nodes need not be identical. Vehicles with different capacities can assure the transport of goods. The objective is to minimize the total transportation cost. The column generation method is used for selecting routes in the mathematical model.

In the paper, no time windows and no conditions for order of pickup and delivery are considered. All the vehicles have the same capacity. Each route has to be cyclical for all the included nodes. Two models are proposed in the paper; the first one is based on the optimal flow theory, the second one uses a model of set covering problem.

Let a distribution network be given by $G=\{V, E]$, where $V$ is a set of $n$ nodes and $E$ is a set of undirected arcs. Each $\operatorname{arc}(i, j)$ is evaluated by distance $c_{i j}$. Let us denote $q_{k l}$ the amount of goods that has to be transported from the node $k$ to node $l$. Vehicles with the capacity $V$ are used for pickup and delivery and they can start in any node. All the routes have to be cyclical, each vehicle has to come back to the node it starts from. The objective is to minimize the length of all the routes.

\section{Example}

Let us consider the distribution network with 4 nodes; the distance matrix $C$ and the transportation requirements matrix $Q$ are given:

$$
C=\left[\begin{array}{l}
0,30,40,60 \\
30,0,60,50 \\
40,60,0,20 \\
60,50,20,0
\end{array}\right] \quad Q=\left[\begin{array}{l}
0,0,7,5 \\
0,0,5,7 \\
5,6,0,0 \\
8,0,8,0
\end{array}\right] .
$$

Vehicle capacity is $V=12$.

\section{Optimal multi-product flow model}

Let us define two additional nodes in the network: the node 0 as the source and the node $n+1$ as the sink. The following variables are defined in the model:

$y_{i j} \geq 0$, integer - number of vehicles going through the arc $(i, j)$ in the direction from $i$ to $j(i, j=0,1, \ldots, n+1, i \neq j)$,

$x_{i j}^{k l} \geq 0$, amount of goods (part of the total amount $q_{k l}$ ) transported from the node $i$ to the node $j(i, j=0,1, \ldots, n+1$, $i \neq j ; k, l=1,2, \ldots, n, k \neq l$ ).

The mathematical model follows:

$$
\begin{aligned}
& z=\sum_{i=1}^{n} \sum_{j=1}^{n} c_{i j} y_{i j} \longrightarrow \min \\
& \sum_{i=1}^{n} y_{i j}=\sum_{i=1}^{n} y_{j i} j=1,2, \ldots, n,
\end{aligned}
$$

\footnotetext{
* Jan Pelikan, Jan Fabry

University of Economics Prague, Czech Republic, E-mail: Pelikan@vse.cz
} 


$$
\begin{aligned}
& \sum_{i=0}^{n} x_{i j}^{k l}=\sum_{i=1}^{n+1} x_{j i}^{k l} j=1,2, \ldots, n, k, l=1,2, \ldots, n, k \neq l \\
& \sum_{k, l} x_{i j}^{k l} \leq V y_{i j}, i, j=1,2, \ldots, n, i \neq j \\
& x_{0, k}^{k l}=q_{k l}, k, l=1,2, \ldots, n, k \neq l ; \\
& x_{0, j}^{k l}=0, k, l=1,2, \ldots, n, k \neq l ; j=1,2, \ldots, n+1, j \neq k, \\
& x_{l, n+1}^{k l}=q_{k l}, k, l=1,2, \ldots, n, k \neq l ; \\
& x_{j, n+1}^{k l}=0, k, l=1,2, \ldots, n, k \neq l ; j=0,1, \ldots, n+1, j \neq k, \\
& x_{i j}^{k l} \geq 0, i, j=0,1, \ldots, n+1, i \neq j ; k, l=1,2, \ldots, n, k \neq l, \\
& y_{k l} \geq 0, \text { integers, } k, l=1,2, \ldots, n, k \neq l
\end{aligned}
$$

The objective (1) corresponds to the sum of the evaluations of all the arcs in the solution, i.e. the total length of all the routes. Equations (2) assure the vehicle will leave the location that it will visit. With respect to equations (3), amount of goods being transported from $k$ to $l$ entering the node $j$ leaves this node. Inequalities (4) disable exceeding the capacity of the vehicle transporting goods between the nodes $i$ and $j$. Equations (5) assure that both the total flow from the source 0 to the node $k$ and the total flow from the node $l$ to the sink $n+1$ are equal to the total requirement $q_{k l}$. All other flows from the source and to the sink are set to 0 .

Example 1:

The application of the model (1) - (6) to the example introduced above leads to the objective value equal to 260 and to the following values of variables:

$y_{12}=y_{13}=y_{24}=y_{34}=1, y_{31}=y_{43}=2($ see Table 1$)$.

Hence, two routes are generated:

route A: $2 \rightarrow 4 \rightarrow 3 \rightarrow 1 \rightarrow 2$ of the length 140

and route $\mathrm{B}: 1 \rightarrow 3 \rightarrow 4 \rightarrow 1$ of the length 120 .

Optimal solution of example 1

Tab. 1

\begin{tabular}{|l|l|l|l|l|l|}
\hline \multicolumn{2}{|l|}{ Route A } & \multicolumn{3}{l|}{ Route B } \\
\hline Node & Pickup & Delivery & Node & Pickup & Delivery \\
\hline 2 & $q_{24}=7 \quad q_{23}=5$ & & 1 & $q_{14}=5 \quad q_{13}=7$ & \\
\hline 4 & & $q_{24}=7$ & 3 & & $q_{13}=7$ \\
\hline 3 & $q_{31}=5 q_{32}=6$ & $q_{23}=5$ & 4 & $q_{41}=8$ & $q_{14}=5$ \\
\hline 1 & & $q_{31}=5$ & 1 & & $q_{41}=8$ \\
\hline 2 & & $q_{32}=6$ & & & \\
\hline
\end{tabular}

Note: The values of matrix $Y$ provide information about the arcs that are included in the optimal routes. Generation of the routes based on this information need not be unique. In addition it is necessary to determine the depot for each route.

\section{Routes generation model}

The model is based on the assumption there are proposed routes satisfying all the conditions of transportation, depots in all the proposed routes are determined. The goal is to select routes satisfying requirements given by the matrix $Q$ and minimizing the total length of all the routes derived from the matrix $C$. A number of vehicles realizing the transport will be determined as well.

Let us assume $S$ routes including arcs of a distribution network, the length of each route is denoted $d s(s=1,2, \ldots, S)$. Parameter $a_{i j}^{k l}(s)$ equals 1 if goods transported on the route $s$ from the node $k$ to node $l$ use the $\operatorname{arc}(i, j), 0$ otherwise.

Example 2:

Parameters $a_{i j}^{k l}(s)$ for the route A: $2 \rightarrow 4 \rightarrow 3 \rightarrow 1 \rightarrow 2$ presented in the previous chapter are defined in Table 2.

Parameters $a_{i j}^{k l}(s)$ for route A

Tab. 2

\begin{tabular}{|c|c|c|c|c|c|c|}
\hline$(i, j) /(k, l)$ & 2.4 & 2.3 & 4.3 & 4.1 & 3.1 & 3.2 \\
\hline$(2,4)$ & 1 & 1 & & & & \\
\hline$(4,3)$ & & 1 & 1 & 1 & & \\
\hline$(3,1)$ & & & & 1 & 1 & 1 \\
\hline$(1,2)$ & & & & & & 1 \\
\hline
\end{tabular}

In the model, the following variables are used:

$y_{s} \geq 0$, integer - number of vehicles on the route $s(s=1,2, \ldots$, $\mathrm{S})$,

$x_{k l}^{s} \geq 0$, amount of goods (part of the amount $q_{k l}$ ) transported on the route $s(k, l=1,2, \ldots, n, k \neq l, s=1,2, \ldots, S)$.

The mathematical model is:

$z=\sum_{s=1}^{s} y_{s} d_{s} \longrightarrow \min$

$\sum_{s=1}^{s} x_{k l}^{s}=q_{k l}, k, l=1,2, \ldots, n, k \neq l$,

$\sum_{k, l} a_{i j}^{k l} x_{k l}^{s} \leq V y_{s}, i, j=1,2, \ldots, n, i \neq j, s=1,2, \ldots, S$,

$x_{k l}^{s} \geq 0, k, l=1,2, \ldots, n, k \neq l, y_{s} \geq 0$, integer,

$s=1,2, \ldots, S$.

The objective function (7) corresponds to the total length of all the routes. Equations (8) assure transport of required amount of goods $q_{k l}$ from the node $k$ to node $l$. Inequalities (9) disable exceeding the capacity of the vehicle transporting goods on the 
route $s$ between the nodes $i$ and $j$. The key issue in this mathematical model is generation of the routes and their number. The same routes with the different depots are considered as different routes in this model.

\section{Case study and conclusions}

The logistic company operating in the Czech Republic solves the problem of routes design. Eight big cities (hubs or transit places) are given, distances of the edges, estimation of future transport requirements and capacities of vehicles are known. Multi-product flow model was used for optimization of routes design. The model contains 30 integer variables and 2100 continuous variables. The computation took 1,5 hour with using CPLEX 10.
Values of $y_{i j}$ give the numbers of vehicles going on the edge $(i, j)$. They were used as the proposal for the optimal design of the routes and determination of depots. Results will bring remarkable decrease of the transportation cost.

In the article, new type of pickup and delivery problem is presented. The first model is based on multi-product flow formulation and enables transit between routes. In the second model, the generated routes are chosen without the possibility of transits.

\section{Acknowledgment}

This research was supported by GACR grant No. GACR 402/09/0041 and GACR402/06/0123.

\section{References}

[1] SAVELSBergh, M., P., M., SOL., M.: The General Pickup and Delivery Problem, Transportation Res. 29, 1995, pp.17-29.

[2] HANG, X., ZHI-LONG, CH., RAJAGOPAL, S., ARUNAPURAM, S.: Solving a Practical Pickup and Delivery Problem, Transportation science, vol. 37, 3/2003, pp. 347-364.

[3] PELIKAN, J.: Discrete Models in Operating Research (in Czech), Professional Publishing.2001, ISBN 80-86419-17-7. 